\title{
Electric and magnetic dipole coupling in near-infrared split ring metamaterial arrays
}

\author{
Ivana Sersic.* Martin Frimmer, Ewold Verhagen, and A. Femius Koenderink \\ Center for Nanophotonics, FOM Institute for Atomic and Molecular Physics (AMOLF), \\ Science Park 102, 1098 XG Amsterdam, The Netherlands \\ (Dated: Submitted to Phys. Rev. Lett., July 15, 2009, resubmitted October 1, 2009.)
}

\begin{abstract}
We present experimental observations of strong electric and magnetic interactions between split ring resonators (SRRs) in metamaterials. We fabricated near-infrared $(1.4 \mu \mathrm{m})$ planar metamaterials with different inter-SRR spacings along different directions. Our transmission measurements show blueshifts and redshifts of the magnetic resonance, depending on SRR orientation relative to the lattice. The shifts agree well with a simple model with simultaneous magnetic and electric near-field dipole coupling. We also find large broadening of the resonance, accompanied by a decrease in effective cross section per SRR with increasing density. These effects result from superradiant scattering. Our data shed new light on Lorentz-Lorenz approaches to metamaterials.
\end{abstract}

PACS numbers: 42.70.-a, 42.25.-p, 78.20.Ci

Since the seminal work of Veselago and Pendry [1], many experimentalists have started to pursue optical materials with negative permittivity $\epsilon$ and permeability $\mu$ [2]. The key motivation is the prospect of 'transformation optics', which allows arbitrary bending of electromagnetic fields, provided one has full control over $\epsilon$ and $\mu$. Particularly exciting examples are perfect lenses, that allow perfect sub-diffraction focusing [1], and 'cloaks' in which light passes an object without scattering [3]. Full control over $\epsilon$ and $\mu$ requires 'metamaterials' of artificial nano-scatterers with electric and magnetic response, arranged in sub-wavelength arrays. The archetypical building block is the split ring resonator (SRR) consisting of a single cut metal loop with an inductive response. In recent years the field of metamaterials has made tremendous progress in shifting the resonant response from microwave to optical frequencies [4, 5, 6, 7, 8]. An important conceptual question is whether the effective response captured by $\epsilon$ and $\mu$ is influenced by coupling between constituents. Coupling between SRRs in vertical 1D stacks [9, 10] has attracted great attention lately outside the scope of metamaterials, e.g., for magnetic waveguides [9, 11, 12, 13], antennas [14], metamaterial lasers [15], and stereomaterials [16]. Although constituent coupling might be anticipated to affect effective medium parameters [17], measured effective responses have been attributed to single constituents in all experiments on metamaterial arrays to date.

In this Letter we present the first measurements of strong constituent coupling in planar SRR metamaterial arrays. We fabricated and characterized SRR lattices with a magnetic response at $\lambda=1.4 \mu \mathrm{m}[5,6]$ in which we vary the spacing between SRRs along different lattice directions independently. We observe large redshifts and blueshifts in the transmission resonances depending on SRR orientation relative to the lattices. We establish that in-plane electric-electric dipole coupling and out-of-plane magnetic-magnetic dipole coupling are strong competing interactions. We explain the shifts by a quasistatic electric and magnetic dipole coupling model [10], that enables us to determine the magnetic and electric polarizability of SRRs. Finally, we discuss the role of dynamic effects on the metamaterial resonance, which are evident in density- (a)
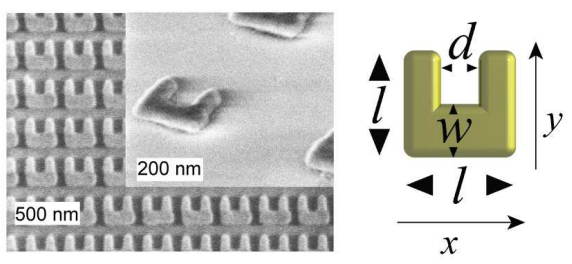

(b)

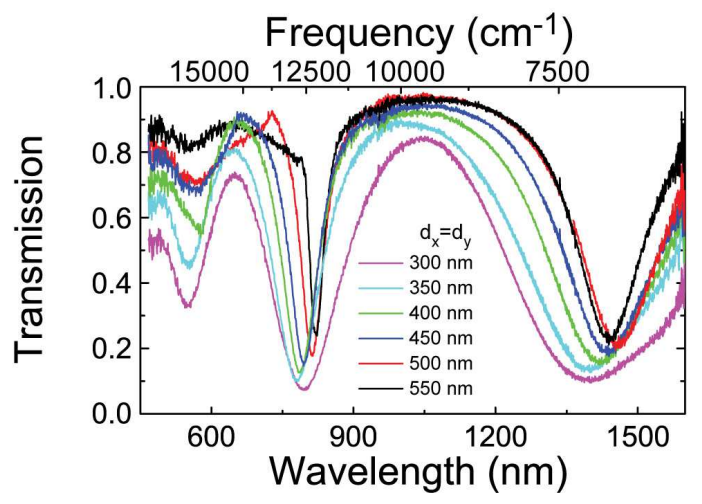

FIG. 1: (Color) (a) We fabricated arrays of Au SRRs on glass with periodicities $d_{x, y}=300 \mathrm{~nm}$ (SEM micrograph) and larger (inset). Each SRR has $l=200 \mathrm{~nm}, w=80 \mathrm{~nm}$, and SRR height $30 \mathrm{~nm}$ ( $\pm 5 \mathrm{~nm}$ ). (b) Transmission spectra for square SRR arrays with split width $d=80 \mathrm{~nm}$ (polarization along $x$ ). The magnetic resonance at $1.4 \mu \mathrm{m}$ blue-shifts and broadens with increasing density.

dependent broadening and a saturation of the transmission.

We have fabricated Au SRRs on glass substrates by electron beam lithography and lift-off using PMMA resist [13], without any adhesive layers. We took great care to produce SRRs of identical dimension in arrays of different densities, using image analysis of SEM micrographs (see Fig. 11a)) to overcome proximity effects. Based on [5], our SRRs (200 nm base) are expected to have an LC resonance at $1.4 \mu \mathrm{m}$. Although driven by the electric field [18], we refer to the resonance as 'magnetic', consistent with literature [2]. To resolve the coupling strength between SRRs along the $x$ (along SRR base) and $y$ (along SRR arms) directions separately, we varied the pitches $d_{x}$ and $d_{y}$ independently between $300 \mathrm{~nm}$ and 550 
(a)

(b)

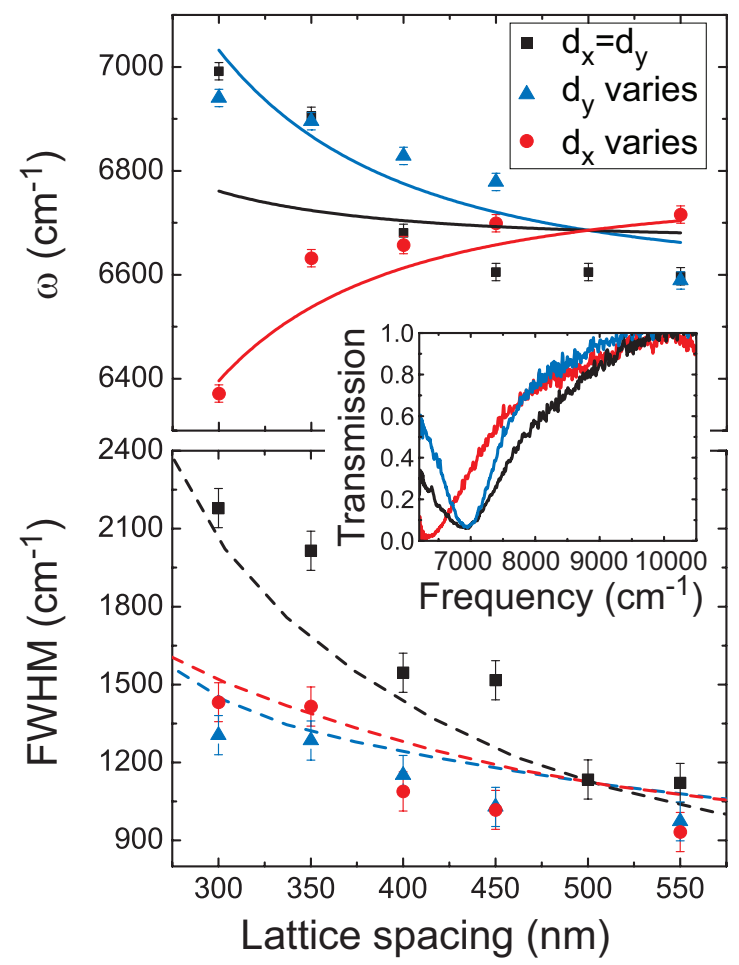

FIG. 2: (Color)(a) Frequency of the magnetic resonance versus lattice spacing. The frequency blue-shifts when decreasing $d_{y}$ whether $d_{y}=d_{x}$ (black squares) or not (blue circles, $d_{x}=500 \mathrm{~nm}$ ), while it red-shifts when decreasing $d_{x}$ (red triangles, $d_{y}=500 \mathrm{~nm}$ ). The inset shows raw spectra for $d_{x}=d_{y}=300 \mathrm{~nm}$ (black curve), $d_{x}=500 \mathrm{~nm}, d_{y}=300 \mathrm{~nm}$ (blue curve), $d_{x}=300 \mathrm{~nm}$, $d_{y}=500 \mathrm{~nm}$ (red curve). (b) FWHM of the magnetic resonance versus lattice spacing (color coding as in (a)). Curves are theory (electrostatic in (a), electrodynamic in (b)).

$\mathrm{nm}$, staying below $550 \mathrm{~nm}$ to avoid grating diffraction in the range of the magnetic resonance. We measured polarizationresolved normal incidence transmission using the set up reported in Ref. [13]. We illuminated a mm-sized area on the sample with a beam from a halogen lamp $\left(5^{\circ}\right.$ opening angle) and used a $20 \mu \mathrm{m}$ pinhole in an intermediate image plane to select the transmitted intensity from single $36 \times 36 \mu \mathrm{m}^{2}$ SRR arrays, which we spectrally resolved by cooled Si CCD and InGaAs array spectrometers, and normalized to transmitted intensity through bare substrate.

Fig. 11 b) shows $x$-polarized transmission spectra measured on a sample with square lattices $\left(d_{x}=d_{y}\right)$ of SRRs with split width $d=80 \mathrm{~nm}$. We observe the magnetic resonance at $1.4 \mu \mathrm{m}$ only for polarization along $x$, as reported by $[5,6,6,18]$, as well as higher order plasmon resonances at $500 \mathrm{~nm}$ and 800 $\mathrm{nm}$ [5]. In this Letter we focus on the magnetic resonance. Tracing the minimum in transmission versus SRR density in Fig. 1 b), we find that the resonance blue-shifts as SRRs are brought closer. A blueshift upon increased coupling is expected by analogy with plasmon hybridization [11, 12, 19], since the magnetic dipoles are all oriented perpendicular to the SRR plane, and hence transversely coupled. To study this cou- pling in detail, we fabricated samples with a large set of SRR arrays (split width $d=100 \mathrm{~nm}$ ) where $d_{x}$ and $d_{y}$ are varied independently. We expect a blueshift with increasing density for all arrays since the magnetic dipoles are always transversely coupled. In Fig.22(a) we plot the measured center frequency of the resonance versus SRR spacing for three sets of arrays. For square lattices $d_{x}=d_{y}$ we indeed observe a continuous blueshift, confirming the data for $d=80 \mathrm{~nm}$ in Fig. 1 b). We also observe a blueshift when only $d_{y}$ is varied $\left(d_{x}=500 \mathrm{~nm}\right)$. Remarkably, we measure a redshift when only $d_{x}$ decreases and $d_{y}$ is fixed at $500 \mathrm{~nm}$. This result is surprising since redshifts imply longitudinal coupling, which is inconsistent with the orientation of the magnetic dipoles. The redshift can only be understood by noting that SRRs also have an electric polarizability in addition to a magnetic dipole [10, 18]. The electric dipole moment points along the SRR base, hence allowing for longitudinal coupling. We implement a model that takes into account simultaneous electric and magnetic dipole coupling, similar to the model for SRR stereodimers reported in [10]. In this model, all magnetic dipoles couple transversely while electric dipoles transversely couple along $y$ and longitudinally along $x$. We limit ourselves to electrostatic and magnetostatic nearest-neighbor coupling, ignoring electro-dynamic effects, the air-glass interface, and multipole corrections. However, this model captures the main physics embodied in our observations. The coupled resonances are set by the Lagrangian

$$
\begin{array}{r}
\mathcal{L}=\sum_{i, j}\left[\frac{L}{2}\left(\dot{Q}_{i, j}^{2}-\omega_{0}^{2} Q_{i, j}^{2}\right)-\frac{M_{h}}{d_{x}^{3}} \dot{Q}_{i, j} \dot{Q}_{i+1, j}\right. \\
\left.-\frac{M_{h}}{d_{y}^{3}} \dot{Q}_{i, j} \dot{Q}_{i, j+1}+2 \frac{M_{e} \omega_{0}^{2}}{d_{x}^{3}} Q_{i, j} Q_{i+1, j}-\frac{M_{e} \omega_{0}^{2}}{d_{y}^{3}} Q_{i, j} Q_{i, j+1}\right],
\end{array}
$$

where $L$ is the SRR inductance, $Q_{i, j}\left(\dot{Q}_{i, j}\right)$ represents the charge (current) on the SRR at site $(i, j)$, and where $M_{h}$ and $M_{e}$ quantify the mutual inductance and the electric dipole coupling. Solving Eq. (1) for the resonance frequency at normal incidence $\left(k_{\|}=0\right)$ yields

$$
\omega=\omega_{0} \sqrt{\frac{1-\frac{4 \kappa_{e}}{d_{x}^{3}}+\frac{2 \kappa_{e}}{d_{y}^{3}}}{1-\frac{2 \kappa_{h}}{d_{x}^{3}}-\frac{2 \kappa_{h}}{d_{y}^{3}}}}
$$

where $\omega_{0}$ is the resonance frequency of a single SRR, and $\kappa_{e, h}=M_{e, h} / L$. Eq. (2) is similar to a prediction by Marqués et al. 17] for 3D SRR arrays. We fix $\kappa_{e}=1.04 \cdot 10^{-21} \mathrm{~m}^{3}$ to match the SRR electric polarizability to the resonant extinction cross section of $0.3 \mu \mathrm{m}^{2}$ measured by Husnik et al. [20, 21], and we set $\kappa_{h}=0.67 \kappa_{e}$. Fig. 3] a) shows the resonance $\omega$ versus $d_{x, y}$ assuming magnetic coupling only ( $\kappa_{e}=$ $0)$. The resonance blue-shifts for decreasing $d_{x, y}$ in all cases due to transverse magnetic dipole coupling. Fig. 3 (b) shows the resonance frequency for electric coupling only $\left(\kappa_{h}=0\right)$. The resonance red-shifts with increasing density unless $d_{x}$ is fixed at $500 \mathrm{~nm}$. This result indicates that longitudinal coupling exceeds transverse coupling in square lattices of strictly 


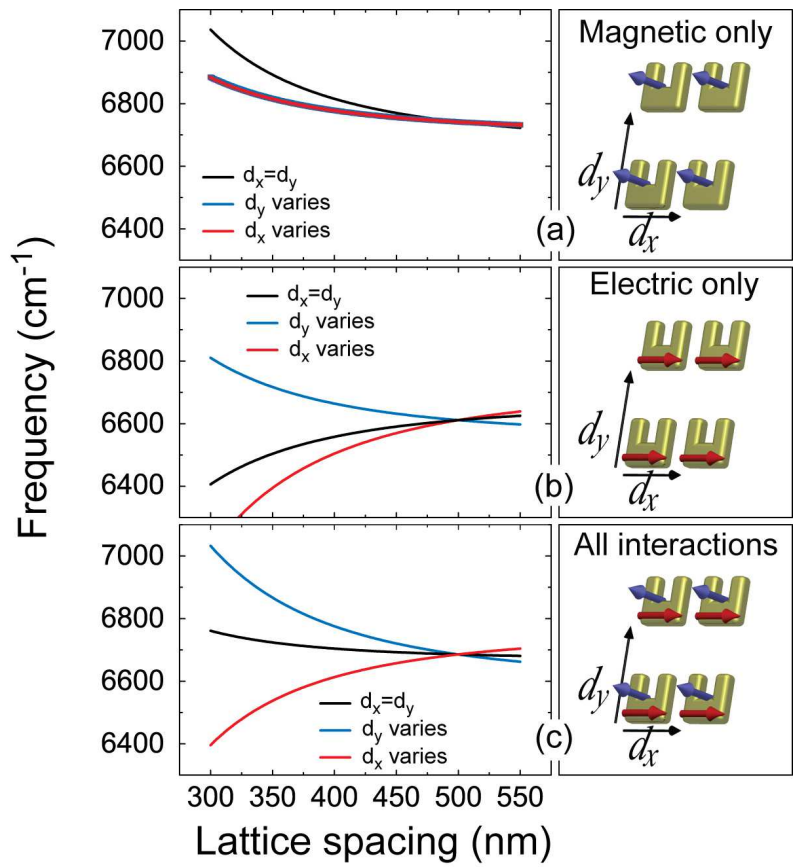

FIG. 3: (Color) Electrostatic calculation of the magnetic resonance frequency as a function of lattice spacing. Black curves: $d_{x}=d_{y}$. Blue curves: $d_{y}$ varies at fixed $d_{x}=500 \mathrm{~nm}$. Red curves: $d_{x}$ varies at fixed $d_{y}=500 \mathrm{~nm}$. For magnetic coupling only (a), resonances always blue-shift with decreasing lattice spacing, while for electric coupling only (b), the behavior of the resonances for $d_{x}=d_{y}$ changes sign with respect to (c) (all couplings). Insets in (a), (b) and (c) are sketches of the electric and magnetic coupling between SRRs. Curves in (c) are reproduced in Fig. 2 ]

in-plane dipoles. This behavior is indeed observed for the purely electric resonance at $800 \mathrm{~nm}$, at least in the regime $\max \left(d_{x}, d_{y}\right) \leq 400 \mathrm{~nm}$ where grating anomalies [22] (asymmetric shoulders at $750 \mathrm{~nm}$ in Fig.1(b)) do not yet set in.

Neither model with solely electric or solely magnetic interaction is consistent with the measured shift of the $1.4 \mu \mathrm{m}$ resonance, since we observe blue-shifts in all cases except when $d_{x}$ is varied and $d_{y}$ is fixed. Fig. 3 ( c) shows the calculated $\omega$ taking into account both electric and magnetic interactions. As in the data, the resonance only red-shifts when decreasing the distance $d_{x}$ at large $d_{y}$. In this case longitudinal electric coupling exceeds the sum of transverse electric and magnetic coupling, leading to a net redshift. For a quantitative comparison with our data we plot the shifts in Fig. 3(c) together with the data in Fig. 2. The good quantitative agreement without any adjustable parameters confirms our interpretation that SRRs in metamaterial arrays show strong electric and magnetic dipole-dipole interactions. These interactions are best quantified in rectangular arrays, since in square arrays studied sofar [5, 6] partial cancellation obscures the magnetically induced blueshift. In our comparison we used $\kappa_{e} / \kappa_{h}=1.5$ measured in [10] for vertically stacked SRRs. The data show that this ratio is also relevant for dipole-dipole coupling in the $x y$-plane, allowing a direct identification of $\kappa_{e}$ and $\kappa_{h}$ with on-resonance electric and magnetic polarizabilities (see note [21]). It is remarkable that the magnetic polarizability $\alpha_{M}$ is of the same order as the electric polarizability $\alpha_{E}$, as opposed to the normal ordering $\alpha_{M} \ll \alpha_{E}$ [23]. This conclusion is in accordance with recent estimates of Merlin [23], that SRRs have $\alpha_{M}$ comparable in magnitude to $\alpha_{E}$ provided $\operatorname{Im} \epsilon_{\mathrm{Au}} \gg \lambda / \ell$, where $\ell$ is the characteristic scatterer size. Given the dielectric constant of $\mathrm{Au} \operatorname{Im} \epsilon_{\mathrm{Au}} \sim 10$, and the size $\lambda / \ell \sim 7$ of our SRRs, their LC resonances are indeed expected to be magnetic resonances with large $\alpha_{M}$. Into the visible, $\operatorname{Im} \epsilon_{\mathrm{Au}}$ rapidly decreases, causing $\alpha_{M}$ to vanish [23], as argued independently in [8].

A striking feature in our transmission data ( $c f$. Fig. 1(b)) in addition to the spectral shifts, is the large broadening of the resonance as the density of SRRs increases. In Fig. 2(b) we plot the measured full width at half minimum (FWHM) of the transmission minimum versus lattice spacing. For square lattices, the width more than doubles from 1000 to $2150 \mathrm{~cm}^{-1}$ as the pitch is reduced from 550 to $300 \mathrm{~nm}$, while for both types of rectangular lattices $\left(d_{x}\right.$ or $d_{y}$ fixed at $\left.500 \mathrm{~nm}\right)$ the width increases from 950 to $1400 \mathrm{~cm}^{-1}$. Such broadening was also noted by Rockstuhl et al. [6] for square arrays. Our extensive data on many rectangular and square arrays allow us to quantitatively identify the source of broadening. From the outset it is clear that the broadening is outside the scope of Eq. (1), since the (Ohmic) damping rate is almost independent of coupling in any electrostatic model. Instead, electrodynamical radiation damping, i.e., scattering loss into the far field must be taken into account. As all oscillators in our sub-diffraction lattices are driven in phase $\left(k_{\|}=0\right)$, scattered light radiated by all oscillators interferes destructively for all angles, except along the transmitted and reflected direction. Since the magnetic dipoles are aligned along the incident beam, they do not radiate any amplitude into the $k_{\|}=0$ directions. Hence, all radiation damping is solely due to the induced electric dipoles. For a quantitative analysis we use an electrodynamical model for electric point dipoles with a Lorentzian resonance in $\alpha_{E}$ according to [12, 21, 22], centered at $1.4 \mu \mathrm{m}$ and including the material loss rate of $\mathrm{Au}$, in addition to radiation damping. We evaluate Eqs. $(8,9)$ in Ref. [22] to predict the array transmission. This dynamic model has no adjustable parameters, since the on-resonance polarizability is fixed [21] to match the extinction cross section of single SRRs in [20]. We find a broadening of the collective transmission resonance that quantitatively reproduces the measured broadening with decreasing pitch for all lattices (FWHM curves in Fig. 2 (b)). An important conclusion is that the large width of the magnetic response commonly observed for SRR arrays [4, 5, 6] is not due to intrinsic loss, but is quantitatively consistent with superradiant decay of the electric dipoles. The collective enhancement of the single SRR radiative linewidth, already suspected by [6], implies enhanced scattering and a reduction of the absorption of the array far below the albedo of single SRRs.

Finally we correlate the resonance broadening with the measured transmission $T$ on resonance. Fig. 4 shows the effective extinction cross section derived from our measure- 


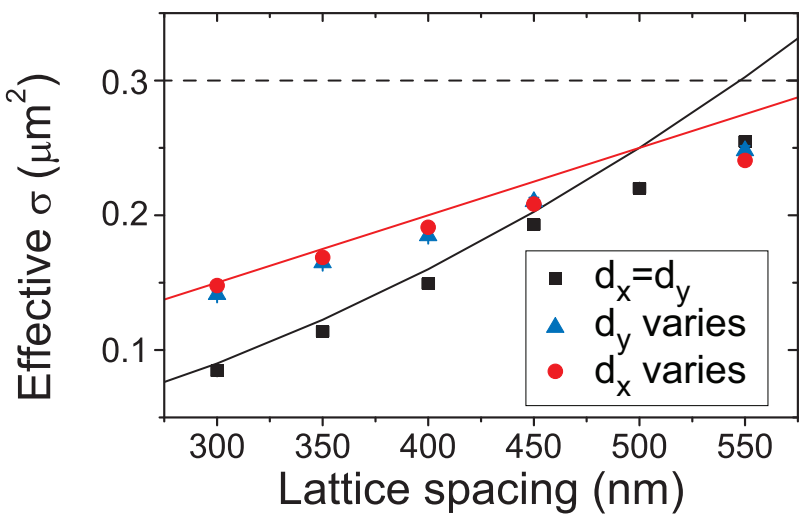

FIG. 4: Effective extinction cross section per SRR derived from onresonance tranmission. The black dashed line indicates the cross section of a single SRR (from [20]). The cross section per SRR is limited by the area $d_{x} d_{y}$ of the unit cell (black and red lines).

ments through $\sigma_{\text {eff }}=d_{x} d_{y}(1-T)$. For uncoupled scatterers we expect constant $\sigma_{\text {eff }}$ equal to the extinction cross section $\sigma_{\text {ext }}=0.3 \mu \mathrm{m}^{2}$ measured for a single SRR in [20] (dashed line in Fig. 4), as indeed almost found in our data for $d_{x}=d_{y}>500 \mathrm{~nm}$. For $d<500 \mathrm{~nm}$, we measure values for $\sigma_{\text {eff }}$ far below $\sigma_{\text {ext }}$ indicative of strong dipole-dipole coupling. The collective superradiant decay (Fig.2(b)) which widens the resonance reduces the extinction per element to remain below the unit-cell area $d_{x} d_{y}$ (curves in Fig. 4).

In conclusion, we have measured large resonance shifts as a function of density in SRR arrays resonant at $\lambda=1.4 \mu \mathrm{m}$. These shifts are due to strong near-field electrostatic and magnetostatic dipole coupling. Furthermore, we observe electrodynamic superradiant damping that causes resonance broadening and an effective reduction of the extinction cross section per SRR. Since the data show that the response of SRR arrays is not simply given by the product of the density and polarizability of single constituents, we conclude that a Lorentz-Lorenz analysis to explain effective media parameters of metamaterials 'atomistically' is not valid [24]. The fact that the Lorentz-Lorenz picture is invalid has important repercussions: It calls for a shift away from the paradigm that the highest polarizability per constituent is required to obtain the strongest electric or magnetic response from arrays of electric or magnetic scatterers. Our experiments show that increasing the density of highly polarizable constituents to raise the effective medium response [5] is ineffective, since superradiant damping limits the achievable response. To strengthen $\epsilon$ or $\mu$, we propose that one ideally finds constituents that have both a smaller footprint and a smaller polarizability per constituent. We stress that even if constituent coupling modifies $\epsilon$ and $\mu$, we do not call into question reported effective medium parameters or the conceptual validity thereof per se. The effective medium regime only breaks down when constituent coupling is so strong that collective modes of differently shaped macroscopic objects carved from the same SRR array have very different resonance frequencies or widths. In this regime inter- esting physics comes into view, particularly regarding active devices. Specific examples are array antennas for spontaneous emission [14] and 'lasing spasers' [15], where the lowest-loss array mode will lase most easily.

We thank Chris Rétif, Dries van Oosten, Jaime Gómez Rivas, Albert Polman and Kobus Kuipers for assistance and discussions. This work is part of the research program of the "Stichting voor Fundamenteel Onderzoek der Materie (FOM)," which is financially supported by the "Nederlandse Organisatie voor Wetenschappelijk Onderzoek (NWO)."

* Electronic address: i.sersic@amolf.nl

[1] V. G. Veselago, Sov.Phys. USPEKHI 10, 509-514 (1968); J. B. Pendry, Phys. Rev. Lett. 85, 3966 (2000).

[2] J. B. Pendry, Physics World 14, 47 (2001); C. M. Soukoulis, S. Linden, and M. Wegener, Science 315, 47 (2007); V. M. Shalaev, Nature Photonics 1, 41 (2007).

[3] U. Leonhardt, Science 312,1777 (2006); J. B. Pendry, D. Schurig, and D. R. Smith, ibid, 1780 (2006).

[4] D. R. Smith et al., Phys. Rev. Lett. 84, 4184 (2000); W. J. Padilla et al., ibid. 96, 107401 (2006); S. Linden et al., Science 306, 1351 (2004).

[5] C. Enkrich et al., Phys. Rev. Lett. 95, 203901 (2005).

[6] C. Rockstuhl et al., Appl. Phys. B 84, 219 (2006).

[7] A. N. Grigorenko et al., Nature 438, 335 (2005); V. M. Shalaev et al., Opt. Lett. 30, 3356 (2005); G. Dolling, M. Wegener, C. M. Soukoulis, and S. Linden, Opt. Lett. 32, 53 (2007).

[8] M. W. Klein et al., Opt. Lett. 31, 1259 (2006).

[9] E. Shamonina, V. A. Kalinin, K. H. Ringhofer, and L. Solymar, J. Appl. Phys 92, 6252 (2002).

[10] N. Liu, H. Liu, S. N. Zhu, and H. Giessen, Nature Photonics 3, 157 (2009).

[11] M. L. Brongersma, J. W. Hartman, and H. A. Atwater, Phys. Rev. B 62, R16356 (2000).

[12] A. F. Koenderink and A. Polman, Phys. Rev. B 74, 033402 (2006).

[13] A. F. Koenderink, R. de Waele, J. C. Prangsma, and A. Polman, Phys. Rev. B 76, 201403R (2007).

[14] J. Li and N. Engheta, Phys. Rev. B 74, 115125 (2006).

[15] N. I. Zheludev, S. L. Prosvirnin, N. Papasimakis, and V. A. Fedotov, Nature Photonics 2, 351 (2008).

[16] S. Zhang et al., Phys. Rev. Lett. 102, 023901 (2009); E. Plum et al., Phys. Rev. B 79, 035407 (2009).

[17] R. Marqués, F. Martin, and M. Sorolla, "Metamaterials with Negative Parameters: Theory, Design, and Microwave Applications," (Wiley, Hoboken NJ, 2008).

[18] N. Katsarakis et al., Appl. Phys. Lett. 84, 2943 (2004).; T. Koschny, M. Kafesaki, E. N. Economou, and C. M. Soukoulis, Phys. Rev. Lett. 93, 10 (2004).

[19] E. Prodan, C. Radloff, N. J. Halas, and P. Nordlander, Science 302, 419 (2003).

[20] M. Husnik et al., Nature Photonics 2, 614 (2008).

[21] We use $\sigma_{\text {ext }}=4 \pi k \operatorname{Im} \alpha$ with $k=2 \pi / \lambda$, using the Lorentzian electric polarizability $\alpha_{0}=\omega_{0}^{2} \kappa_{e} /\left(\omega_{0}^{2}-\omega^{2}-i \omega \gamma\right)$ implicit in Eq. [2], and with radiation damping $1 / \alpha=1 / \alpha_{0}-i \frac{2}{3} k^{3}$ [12]. We take $\gamma=1.2 \cdot 10^{14} \mathrm{~s}^{-1}$ for the Ohmic damping of Au from P. B. Johnson and R. W. Christy, Phys. Rev. B. 6, 4370 (1972).

[22] F. J. García de Abajo, Rev. Mod. Phys. 79, 1267 (2007).

[23] R. Merlin, Proc. Natl. Acad. Sci. U.S.A. 106, 6 (2009). 
[24] C. R. Simovski and S. A. Tretyakov, Phys. Rev. B 75, 195111 (2007). 